K.A. van der Hucht, G. Koenigsberger \& P.R.J. Eenens, eds.

\title{
Thermal instability in the wind of Wolf-Rayet stars
}

\author{
Denise R. Gonçalves \\ Instituto de Astrofísica de Canarias, E-38200 La Laguna, Tenerife, \\ Spain
}

\author{
Vera Jatenco-Pereira and Reuven Opher \\ Universidade de São Paulo, Instituto Astronômico e Geofísico, \\ Av. Miguel Stéfano, 4200, 04301-904, São Paulo, Brazil
}

\begin{abstract}
In this study, we investigate the formation of clumps at the base of the wind of early-type stars via thermal instability in the presence of Alfvén wave heating. As a result we show the existence of two stable equilibrium regions, one at high temperature, the diffuse medium, and another at low temperature, the clumps. We show that the two stable equilibrium regions can coexist over a narrow range of pressures describing the diffuse medium and the clumps.
\end{abstract}

\section{Introduction}

The observed IR emission of Wolf-Rayet stars is often stronger than expected from a homogeneous wind. The cause may be that clumping in the stellar wind increases the mean density and hence the emission measure. Clumping can also explain the observed enhancement in the blue wing of some observed lines. The narrow absorption components are likely to be direct manifestations of dense clumps.

We study here a mechanism to form condensations in the base of WR stars winds. That means: we study the relevance of a flux of Alfvén waves in the hot medium present in the base of WR winds, in order to understand the formation of the clumping features.

The basis for this approach is the work of dos Santos et al. (1993) - a wind acceleration model for WR stars where Alfvén waves act jointly with the radiation pressure. We consider a thin hot corona atmosphere, which corresponds to the X-rays observed, with a temperature of $\sim 10^{7} \mathrm{~K}$, and density of $\sim 2 \times 10^{13} \mathrm{~cm}^{-3}$ (see van der Hucht 1992). Our final goal is to demonstrate the stability of the base of the wind that has 'cool' $\left(\sim 10^{4} \mathrm{~K}\right)$ clouds and a 'hot' $\left(\sim 10^{7} \mathrm{~K}\right)$ intercloud medium coexisting at the same pressure.

\section{The model}

The physical processes on the heating-loss function $H(T, n)$ include: losses via Bremsstrahlung, $H_{b}$; an approximate expression to express the residual heating due to radiative ionization followed by recombination, $H_{r}$; a fit to radiative losses in the range $10^{4} \mathrm{~K}$ and $10^{8} \mathrm{~K}$ due to electron excitation of resonance transitions in 
common metal ions is, $H_{r l}$ (Mathews \& Doane 1990); Compton heating-cooling, $H_{c}$; and Alfvén wave heating, $H_{a}$.

There are in the literature several damping mechanisms for Alfvén waves. Here we discuss two of them: nonlinear and turbulent damping.

The complete heating-cooling function, $H(T, n)$, including the physical processes above, is: $H(T, n)=H_{b}+H_{r}+H_{r l}+H_{c}+H_{a}$.

As we are forming clouds, via thermal instability, from the hot atmosphere, we performed calculations in order to find, for each temperature, the density that corresponds to the balance $(H(n, T) \approx 0)$. From the isobaric instability's criterion, we need clouds and hot medium co-existing at the same pressure. In the hot atmosphere this pressure is $P=2 n_{\mathrm{H}} k T_{\mathrm{H}} \approx 5.5 \times 10^{4} \mathrm{dyn}_{\mathrm{cm}}^{-2}$.

\section{Discussion}

The nonlinear and turbulent Alfvenic heatings represent extreme opposite dependencies of these heatings on density. Turbulent heating $\left(H_{T} \propto n^{7 / 12}\right)$ deposits more energy at the center of the cloud, where the density is higher. Nonlinear heating $\left(H_{T} \propto n^{-1 / 2}\right)$ deposits more energy in the outer regions of the clouds, where the density is lower. Due to the completely different behavior of these heatings, the results from each one are very different. In addition, the low temperature solution reached by each of these heatings is different.

Any thermal instability of the wind material would have to compete against the intrinsic line-driven instability of the flow. Then, consider that the cooling time or thermal instability time in an unperturbed medium is $t_{\text {cool }} \approx \frac{k_{B} T}{n \Delta} \simeq 1.6 \mathrm{~s}$ (at $P_{\mathrm{H}}=5.5 \times 10^{4} \mathrm{dyn} \mathrm{cm}^{-2}$ ) where $T \equiv T_{\mathrm{H}}, k_{B}$ is the Boltzman constant, and $\Delta$ is the cooling rate dominated by radiative processes $\left(H_{b}+H_{r l}\right)$. Consider also that the dynamical time, or the line-driven instability time, at the base of the wind (up to one stellar radius), is $t_{\mathrm{dyn}} \approx \frac{R}{\langle v\rangle} \simeq 1.6 \times 10^{3} \mathrm{~s}$, where $R$ is the distance from the ionization source $\left(\sim 0.1 R_{*}\right)$ and $\langle v\rangle \simeq 5.04 \times 10^{7} \mathrm{~cm} \mathrm{~s}^{-1}$ (Owocki 1994) is the wind velocity in this region. The above estimates show that the cooling time is much smaller than the dynamical time, as is necessary in order to have the thermal instability predominate in this region. See Gonçalves et al. (1998) for a detailed discussion.

Acknowledgments. The authors would like to thank the Brazilian agencies FAPESP, CNPq and Pronex/FINEP (41.96.0908.00) for partial support.

\section{References}

dos Santos, L.C., Jatenco-Pereira, V., Opher, R. 1993, ApJ 410, 732

Gonçalves, D.R., Jatenco-Pereira, V., Opher, R. 1998, ApJ 501, 797

van der Hucht, K.A. 1992, The A\&A Rev 4, 123

Mathews, W.G., Doane, J.S. 1990, ApJ 352, 423

Owocki, S.P. 1994, in: A.F.J. Moffat, S.P. Owocki, A.W. Fullerton \& N. St-Louis (eds.), Instability and Variability of Hot-Star Winds, Proc. Isle-aux-Coudres Workshop 1993, ApSS 221, 3 